\title{
Stellar Crowding and the Science Case for Extremely Large Telescopes
}

\author{
Knut A.G. Olsen \\ National Optical Astronomy Observatory, CTIO, Casilla 603, La Serena, Chile \\ kolsen@noao.edu \\ Robert D. Blum \\ National Optical Astronomy Observatory, CTIO, Casilla 603, La Serena, Chile \\ rblum@noao.edu \\ François Rigaut \\ Gemini Observatory, 670 N. A'ohoku Place, Hilo, Hawaii, 96720, USA \\ frigaut@gemini.edu
}

\begin{abstract}
We present a study of the effect of crowding on stellar photometry. We develop an analytical model through which we are able to predict the error in magnitude and color for a given star for any combination of telescope resolution, stellar luminosity function, background surface brightness, and distance. We test our predictions with Monte Carlo simulations of the LMC globular cluster NGC 1835, for resolutions corresponding to a seeing-limited telescope, the HST, and an AO-corrected 30-m (near diffraction limited) telescope. Our analytically predicted magnitude errors agree with the simulation results to within $\sim 20 \%$. The analytical model also predicts that errors in color are strongly affected by the correlation of crowding-induced photometric errors between bands as is seen in the simulations. Using additional Monte Carlo simulations and our analytical crowding model, we investigate the photometric accuracy which 30-m and 100-m Extremely Large Telescopes (ELTs) will be able to achieve at distances extending to the Virgo cluster. We argue that for stellar populations work, ELTs quickly become crowding-limited, suggesting that low-Strehl AO systems may be sufficient for this type of science.
\end{abstract}

Subject headings: techniques: photometric — galaxies: stellar content

\section{Introduction}

The study of stellar populations in nearby galaxies has benefited tremendously from the high sensitivity and resolution of modern ground- and space-based telescopes. Nevertheless, our ability to study the evolutionary histories of a statistical sample of galaxies through the properties of their 
resolved stars is severely hampered by insufficient resolution to overcome the effects of crowding. For the study of the oldest ( 13 Gyr) main sequence stars, even the Hubble Space Telescope is limited to the nearest dwarf companions to the Milky Way, while at the distance of M31 only the brightest stars or those lying in the lowest surface brightness regions are resolved.

The 30-100m Extremely Large Telescopes (ELTs) now being considered ${ }^{1}$ will provide a giant leap in our ability to study stellar populations in nearby galaxies. Using adaptive optics (AO) to correct for the wavefront distortion produced by the Earth's atmosphere, these telescopes aim to deliver near diffraction-limited performance at wavelengths of approximately one to two microns. Such resolution will vastly improve our ability to observe faint stars projected against dense backgrounds. Nevertheless, even ELTs will be crowding-limited in many cases.

In this paper, we study the impact of crowding on the stellar populations science case of ELTs. The effects of crowding have been extensively studied by both the optical and radio astronomy communities, using both analytical and numerical methods. These studies have addressed the photometric errors, incompleteness, and positional errors introduced by crowding (e.g., Scheuer 1957, Condon 1974, Gallart, Aparicio, \& Vílchez 1996, Renzini 1998, Hogg 2001, Stephens et al. 2001). This paper by no means supersedes this previous work, which as a whole forms an almost complete study of the subject. Instead, our goal is to develop a simple set of tools to help assess the stellar populations science that may be done with ELTs, focussing particularly on the issue of crowding-induced photometric errors. We hope that these tools may also be useful to a broader set of applications.

In section 2, we develop a simple analytical model of the photometric effects of crowding. We test the model against Monte Carlo simulations ("artificial star tests") in section 3. In section 4, we use further Monte Carlo simulations to demonstrate the performance of the proposed 30-m Giant Segmented Mirror Telescope (GSMT) in crowded Local Group environments. We use our analytical model to determine the distances out to which 30-m and 100-m AO-corrected telescopes could perform useful photometry in section 5. Section 6 contains our summary and conclusions.

\section{An Analytical Model of Crowding}

For evaluating the possibility of studying stellar populations in distant galaxies with ELTs, we are particularly interested in answering the question, "Given an ELT, to what distance and background surface brightness can we measure the colors and magnitudes of stars with $x \%$ accuracy?" We will thus mainly consider the photometric effects of crowding. We will approach the problem by considering the fluctuations in luminosity produced by a stellar background, as was done by

\footnotetext{
${ }^{1}$ e.g. the Giant Segmented Mirror Telescope (http://www. aura-nio.noao.edu/), the California Extremely Large Telescope (http://celt.ucolick.org/), the OWL telescope (http://www.eso.org/projects/owl/), the Euro50 telescope (http://www.astro.lu.se/ torben/euro50/)
} 
Tonry \& Schneider (1988) for the case of distant, unresolved stellar populations. We note that for the purposes of this study, we ignore all other sources of photometric error, e.g. shot noise, read noise, and flat-fielding errors. For the environments studied here, crowding is typically the dominant source of error.

A basic limitation of stellar photometry is that there is no way to remove the star of interest and measure the contribution to its luminosity of the background underneath. Instead, the background must be estimated from adjacent resolution elements, either by averaging the luminosity within an annulus or by fitting a surface to the surrounding light. Thus, the accuracy with which the background can be estimated (hence the accuracy with which we can measure the light from the star) is limited by the size of random luminosity fluctuations within a single resolution element. By calculating the typical amplitude of these fluctuations, we can study quantitatively the effect of crowding on photometry.

If we are given a luminosity function (LF) $\Phi(l)$ such that $d N=A \Phi(l) d l$, where $d N$ is the number of stars with luminosity between $l$ and $l+d l$ and $A$ is the normalization, then from Poisson statistics the fluctuation in the luminosity due to shot noise in the number of stars in the interval $l, l+d l$ is

$$
\sigma_{l}=l \sigma_{N}=l \sqrt{A \Phi(l) d l}
$$

While performing stellar photometry, either through fixed apertures or through profile fits, we imagine starting with the brightest stars and "peeling away" increasingly fainter ones. Thus, we consider that the background underneath a star of luminosity $L$ in the image receives contributions only from stars with luminosities $l<L$. We calculate the typical background fluctuation by summing over the contributions of these fainter stars. In the limit as the width of the interval $d l$ goes to zero, we find:

$$
\sigma_{L}=\lim _{d l \rightarrow 0} \sqrt{\sum \sigma_{l}^{2}}=\sqrt{A \int_{0}^{L} l^{2} \Phi(l) d l}
$$

Now we need to express $A$ in terms of measurable quantities. The total background luminosity within the average resolution element is:

$$
\Sigma_{\mathrm{res}}=A \int_{0}^{\infty} l \Phi(l) d l
$$

If we make the simplifying assumption that only sources within the circular angular resolution element with diameter $a_{\text {res }}$ contribute to the light within that element, then the surface luminosity within a $1^{\prime \prime}$ patch of the sky is:

$$
\Sigma_{L}=\frac{4 \Sigma_{\mathrm{res}}}{\pi a_{\mathrm{res}}^{2}}
$$

This is equivalent to assuming a "top-hat" point spread function. We then express $A$ in terms of the surface luminosity, the angle $a_{\text {res }}$, and the first moment of the luminosity function using equations 3 and 4 :

$$
A=\frac{\pi \Sigma_{L} a_{r e s}^{2}}{4 \int_{0}^{\infty} l \Phi(l) d l}
$$


Now we can phrase in mathematical terms the question posed above in words. We say that, given no other source of errors, confusion-limited photometry of a star with luminosity $L$ requiring fractional accuracy $x$ is constrained by the inequality:

$$
\frac{\sigma_{L}}{L}<x
$$

Using equation 2 and subsituting the result for $A$ given by equation 5 , we find

$$
\frac{\sqrt{\pi \Sigma_{L} a_{\text {res }}^{2} \int_{0}^{L} l^{2} \Phi(l) d l}}{L \sqrt{4 \int_{0}^{\infty} l \Phi(l) d l}}<x
$$

Equation 7 is our basic equation describing the effects of crowding on stellar photometry. We note again that we have assumed a circular "top-hat" PSF in our derivation. A more rigorous approach would be to adopt a realistic PSF and to integrate the contribution of all stars to any given image coordinate, as was done by e.g. Scheuer (1957). However, for the PSFs and LFs used here, the integral quickly converges within a small region surrounding the coordinate of interest; within this region, the light added by PSF wings from stars outside the boundary of the resolution element is compensated by the loss of light through the PSF wings from stars within the resolution element, such that the effect of our assumption of a top-hat PSF is small. We have also implicitly assumed that stellar clustering occurs only at scales larger than the size of our resolution element. In the

case of star clusters in nearby galaxies, this assumption is valid. Binaries, on the other hand, violate this assumption; their effect could instead be included in the stellar luminosity function.

Equation 7 will be easier to use if we express the surface luminosity in apparent magnitudes per square arcsec and the stellar luminosity in absolute magnitudes. Rearranging the equation, we find:

$$
\Sigma_{m}>2 M-2.5 \log \left(\frac{4}{\pi}\left(\frac{\sigma_{m}}{1.086 a_{\mathrm{res}}}\right)^{2} \frac{\int_{M_{l o}}^{M_{h i}} 10^{-0.4 M^{\prime}} \Phi\left(M^{\prime}\right) d M^{\prime}}{\int_{M_{l o}}^{M} 10^{-0.8 M^{\prime}} \Phi\left(M^{\prime}\right) d M^{\prime}}\right)+(m-M) 。
$$

where $\Sigma_{m}$ is the surface brightness of the background in magnitudes per square arcsecond, $M$ is the absolute magnitude of the star we want to measure, $M_{\mathrm{lo}}$ is the absolute magnitude of the faintest star in the LF, $M_{\mathrm{hi}}$ is the absolute magnitude of the brightest star in the LF, $(m-M)_{0}$ is the distance modulus, and $\sigma_{m}$ is the photometric accuracy in magnitudes.

\subsection{Consequences of the crowding model}

Considering the case of a delta-function LF, $\Phi\left(M^{\prime}\right) d M^{\prime}=\delta\left(M^{\prime}-M\right) d M^{\prime}$, equation 8 reduces to:

$$
\Sigma_{m}>M-2.5 \log \left(\frac{4}{\pi}\left(\frac{\sigma}{1.086 a_{\mathrm{res}}}\right)^{2}\right)+(m-M)_{\circ}
$$


which yields the magnitude of the faintest star that can be observed in a region of surface brightness $\Sigma_{m}$ mags $\operatorname{arcsec}^{-2}$ with a given telescope and desired photometric accuracy:

$$
m<\Sigma_{m}-2.5 \log \left(\frac{\pi}{4}\left(\frac{1.086 a_{\mathrm{res}}}{\sigma_{m}}\right)^{2}\right)
$$

If we set $\sigma_{m}=0.2$, then equation 10 is equivalent to the commonly used rule of thumb that photometry becomes confusion-limited when the background surface brightness equals that produced if the light from the star were spread over 30 resolution elements (e.g. Hogg 2001). For more accurate photometry, the confusion limit must be taken to be more conservative.

What is the effect of the shape of the luminosity function on the confusion limit? If we assume a power-law luminosity function $\Phi(l)=l^{\alpha}$, then for bright stars with $M \sim M_{\text {hi }}$, equation 8 becomes:

$$
\Sigma_{m} \gtrsim M-2.5 \log \left(\frac{4}{\pi}\left(\frac{\sigma_{m}}{1.086 a_{\mathrm{res}}}\right)^{2}\right)-2.5 \log \left(\frac{3+\alpha}{2+\alpha}\right)+(m-M) 。
$$

for $\alpha>-2$. Comparing equations 10 and 11, we find that by distributing stars over a range of brightnesses, the surface brightness limit at which one can perform photometry of stars with $M \sim M_{\text {hi }}$ with accuracy $\sigma_{m}$ becomes brighter. That is, given a fixed amount of background light, splitting it up into increasing numbers of fainter stars causes the luminosity fluctuations within a resolution element containing a bright star of interest to decrease. The worst-case scenario is one in which all of the stars have the same brightness as the one we would like to measure, i.e. equation 10. In the solar neighborhood, $\alpha \sim-1.25$ for $0 \lesssim M_{V} \lesssim 12$ (Binney \& Merrifield 1998), so that the term $-2.5 \log \left(\frac{3+\alpha}{2+\alpha}\right) \sim-0.9$ magnitudes.

To explore the effect of the shape of the luminosity function in greater detail, we use equation 8 to examine the effects of age, metallicity, and the slope of the IMF on crowding. Figs. 1-3 show the surface brightnesses at which photometry is limited by crowding to an accuracy of $10 \%$ in $V$ alongside the $V$ model luminosity functions, which were calculated using Girardi et al. (2000) isochrones. We have assumed a distance modulus of $(m-M)_{\circ}=18.5$, a resolution of $1^{\prime \prime}$, and a singleage population for these calculations; the results can be easily translated to other distances simply by adding the difference $(m-M)_{\circ}-18.5$ to the surface brightnesses, and to other resolutions by adding $-2.5 \log \left(a_{\text {res }}^{2}\right)$. Fig. 1 shows the crowding-limiting surface brightnesses for stars with $-3<M_{V}<11$ as a function of age of the environment, assuming solar metallicity, a Salpeter IMF, and a single burst of star formation. For stars below the main sequence turnoff (which produces a break in the LF at $M_{V} \sim 2-4$ ), we find that the limiting surface brightness becomes fainter with increasing age. This is simply the effect of stellar evolution; the number of stars below the turnoff remains unchanged, but the evolution of massive stars causes the environment to dim in brightness. The spacing of the lines for different values of $M_{V}$ is set by the local slope of the LF; at ages between 2 and $4 \mathrm{Gyr}$, the LF is almost flat for $5<M_{V}<3$, and the lines lie very close to each other. This is because the photometric error contributed by crowding is dominated by stars with brightness close to that of the star of interest; thus, a flat LF produces roughly equal crowding errors over a large range in magnitude. Above the main sequence turnoff, the shapes of 
the curves in Fig. 1 are affected strongly by the appearance of the turnoff. The sharp decrease in the LF means that stars brighter than the turnoff may be measured in brighter environments. Finally, stars with $M_{V} \sim-3$ are particularly easy to measure, since the theoretical luminosity functions cut off at $M_{V} \sim-1$. The second term in equation 8 becomes increasingly unimportant, and the crowding-limited surface brightness simply becomes proportional to $2 M_{V}$. This regime is

a bit artificial, however, since normally the star of interest is drawn from the same population as the environment in which it is embedded.

Fig. 2 shows the effect of variations in metallicity on the surface brightness at which one can perform $10 \%$ photometry at a distance modulus of 18.5. In calculating these curves, we have assumed an age of 5 Gyr and a Salpeter IMF. Changing the metallicity has very little effect on the crowding-limited surface brightnesses, except for $M_{V} \sim-3$, near where the theoretical luminosity functions cut off.

Finally, Fig. 3 shows the effect of varying the slope $x$ of the IMF on the crowding-limited surface brightness, where the IMF is given by $d N=A m^{-x} d m$ and $m$ is the stellar mass. For these calculations, we have used an age of 5 Gyr and solar metallicity. Changing the IMF slope has dramatic effect on the surface brightness limits for stars below the main sequence turnoff. This is again caused by the fact that the crowding errors are dominated by fluctuations produced by stars with similar brightness to the one of interest. Carrying this reasoning through with an example, the curve for $M_{V}=9$ in Fig. 3 is roughly defined by keeping the number of stars with $M_{V}=9$ constant as the IMF slope is varied. Making the IMF slope steeper thus decreases the brightness of the environment, while making it flatter increases it, as is seen in the figure. By contrast, for bright stars, we find that steepening the slope of the IMF makes the surface brightness at which we can perform $10 \%$ photometry slightly brighter. This behavior is predicted by equation 11, where we set $M \sim M_{\mathrm{hi}}$.

Figs. 1-3 are useful for estimating the surface brightness above which $10 \% \mathrm{~V}$-band photometry becomes impossible due to crowding. Figs. $4-6$ show similar predictions for $K$-band photometry, again assuming a distance modulus of 18.5 and $1^{\prime \prime}$ resolution.

\subsection{The effect of crowding on colors}

So far, we have considered only the effect of crowding on the measurement of stellar magnitudes. What about the effect on colors? To calculate this, we have to account for the fact that the crowding-induced errors are correlated between bands. That is, given two resolution elements $a_{1}$ and $a_{2}$ with corresponding bands $m_{1}$ and $m_{2}$, many stars contribute light to both $a_{1}$ and $a_{2}$. The crowding-induced variance in the color $m_{1}-m_{2}$ is calculated through the covariance matrix:

$$
\sigma_{m_{1}-m_{2}}^{2}=\sigma_{m_{1}}^{2}+\sigma_{m_{2}}^{2}-2 \sigma_{m_{1} m_{2}}^{2}
$$


where $\sigma_{m_{1}}^{2}$ and $\sigma_{m_{2}}^{2}$ are the variances for bands one and two and $\sigma_{m_{1} m_{2}}^{2}$ is the covariance. The variances $\sigma_{m_{1}}^{2}$ and $\sigma_{m_{2}}^{2}$ are calculated following equations 1 and 2:

$$
\begin{array}{r}
\sigma_{L_{1}}^{2}=A_{1} \int_{0}^{L_{1}} l_{1}^{2} \Phi_{1}\left(l_{1}\right) d l_{1} \\
A_{1}=\frac{\pi \Sigma_{L_{1}} a_{1}^{2}}{4 \int_{0}^{\infty} l \Phi_{1}\left(l_{1}\right) d l_{1}} \\
\sigma_{m_{1}}=1.086 \sigma_{L_{1}} / L_{1} \\
\sigma_{L_{2}}^{2}=A_{2} \int_{0}^{L_{2}} l_{2}^{2} \Phi_{2}\left(l_{2}\right) d l_{2} \\
A_{2}=\frac{\pi \Sigma_{L_{2}} a_{2}^{2}}{4 \int_{0}^{\infty} l \Phi_{2}\left(l_{2}\right) d l_{2}} \\
\sigma_{m_{2}}=1.086 \sigma_{L_{2}} / L_{2}
\end{array}
$$

To calculate the covariance, we take the stars occupying resolution elements $a_{1}$ and $a_{2}$ to follow a bivariate Poisson distribution with means $N_{0}+N_{a}=N_{1}$ and $N_{0}+N_{b}=N_{2}$, where $N_{0}$ is the number of stars in common to $a_{1}$ and $a_{2}$. Then (adopting similar notation as for the single star case) $\sigma_{l_{1} l_{2}}^{2}=A_{12} l_{1} l_{2} N_{0}$. In the case of circular resolution elements, the stars found in the smaller resolution element are completely contained by the larger, and we find:

$$
\begin{array}{r}
\sigma_{L_{1} L_{2}}^{2}=A_{12} \int_{0}^{L_{1}} \int_{0}^{L_{2}} l_{1} l_{2} \Phi_{12}\left(l_{1}, l_{2}\right) d l_{1} d l_{2} \\
A_{12}=\frac{\pi \Sigma_{L_{1}} \min \left(a_{1}^{2}, a_{2}^{2}\right)}{4 \int_{0}^{\infty} \int_{0}^{\infty} l_{1} \Phi_{12}\left(l_{1}, l_{2}\right) d l_{1} d l_{2}} \\
\sigma_{m_{2} m_{2}}=1.086 \sigma_{L_{1} L_{2}} / \sqrt{L_{1} L_{2}}
\end{array}
$$

where we have arbitrarily chosen to consider the crowding-induced color errors as a function of $m_{1}$. Since $\sigma_{m_{1} m_{2}}^{2}$ is always positive, the error in color in the crowding-limited case will always be smaller than the simple sum in quadrature of the individual magnitude errors. If $\sigma_{m_{1} m_{2}}^{2}$ is large enough, then one may be able to measure colors more accurately than individual magnitudes.

\section{The LMC as a Case Study}

Using equations 8 and 12, we can predict the photometric error due to crowding for any given telescope resolution, background surface brightness, distance, and luminosity function. To test these predictions, we conducted simulations of a field in the LMC which contains a globular cluster projected against a dense background of field stars, and compared them to observations of the real LMC globular cluster NGC 1835. In the following section, we demonstrate the effect of crowding in the LMC under natural seeing conditions, at HST resolution, and finally at the resolution of an AO-corrected 30-m GSMT. 


\subsection{The LMC from the ground}

Figure 7 shows a seeing-limited $V$ image of NGC 1835 and its surrounding field. This image was taken by A. Walker as part of an observing run with the CTIO 1.5-m telescope and Tek 2048 CCD, with the original aim of calibrating the HST WFPC2 photometry of Olsen et al. (1998). The field of view of the camera is $8.3 \times 8.3$. The images were taken through CTIO copies of the HST F555W and F814W filters on the nights of 1995 January 23-26 under photometric conditions and in good seeing $\left(\mathrm{FWHM} \sim 1^{\prime \prime}\right)$.

Figure 7 also shows the $V-I, V$ color-magnitude diagram derived from the seeing-limited images, plotted in 4 radial bins centered on NGC 1835. The photometry was performed with DAOPHOT/ALLSTAR (Stetson 1987), as follows. After identifying stars down to a signal-to-noise threshold of $3 \sigma$ and performing aperture photometry out to a diameter of 7 !'2, we derived PSFs using $\sim 200$ bright stars in each image. We then computed PSF magnitudes with ALLSTAR, used these magnitudes to subtract all stars except for the PSF stars from the original images, and rederived the PSFs with the neighbor stars removed. We then recomputed the PSF magnitudes with ALLSTAR, measured aperture corrections using the PSF stars alone, and calibrated the photometry using the transformation equations derived by A. Walker from observations of Landolt (1992) fields and $\omega$ Centaurus.

It is clear from the CMDs in Figure 7 that the photometric error and completeness at a fixed magnitude increase dramatically towards the center of the star cluster, as is to be expected when crowding dominates the photometric errors. In order to quantify the effects of the crowding, we performed simulations using artificial $V$ and $I$ images of the cluster and field. These images were produced by combining two theoretical populations, a 14 Gyr old population with $[\mathrm{Fe} / \mathrm{H}]=-1.5$ representing the cluster, the other population having the NGC 1835 field star formation history derived by Olsen (1999) and the chemical enrichment history assumed therein. We selected the individual stars described by these populations using Girardi et al. (2000) isochrones (which we interpolated in age and metallicity), and assuming a Salpeter (1955) initial mass function. We

sampled stars from the entire mass range available in the isochrones; the lowest mass stars have masses of $0.15 M_{\odot}$ and $M_{V} \sim 11.5$. The number of stars and their assigned positions match our observed $V$ surface brightness profile, which we parameterized using a King (1966) model having a 1". 8 core radius and concentration $\left(\log \left(r_{t} / r_{c}\right)\right) 1.7$ plus a constant background. We added the stars to the artifical images using the DAOPHOT routine ADDSTAR and the PSFs derived from the true images, and assuming an LMC distance modulus of 18.5. The full artificial images, of which we produced one each in $V$ and $I$, contain $>5 \times 10^{6}$ stars down to $V \sim 30$ and include stars with the lowest mass available in the Girardi et al. (2000) isochrones, $0.15 M_{\odot}$. In order to improve the statistics in the highest surface brightness regions, we simulated an additional 100 images in $V$ and $I$ of the central $72^{\prime \prime}$. These images are slightly shallower, with $>4 \times 10^{5}$ stars down to $V \sim 28$, but still contain more than $95 \%$ of the light of the same region in the deeper image. We used these images in our analysis of the photometric errors in the central $72^{\prime \prime}$. 
We performed photometry on the artificial images exactly as we did for the 1.5-m images. Figure 8 shows the CMD obtained from one of the pairs of deep artificial images alongside the artificial $V$ image itself. While there are differences in detail between the artifical and observed images and CMDs, the simulation reproduces the basic features of the observations: the size and shape of the cluster, the field star density, and the broad features of the CMD. We thus conclude that the simulation is adequate for a realistic study of crowding in this field.

We identified which of the stars in the input list were recovered by the simulations by comparing the input and output lists by $\mathrm{x}$ and $\mathrm{y}$ position. We found that $99 \%$ of the recoveries were found within 2 pixels $\left(\sim 0^{\prime \prime} 5\right.$, or one-half times the FWHM) of their input position, so we adopted this as the radius beyond which we considered input stars lost during the simulation. In cases where multiple matches were found within 2 pixels, we chose the pair with the smallest absolute difference in magnitude. We rejected stars with input magnitudes $>22.5$, as these appeared to produce only spurious matches. For the same reason, we also rejected stars which were recovered more than two magnitudes brighter than their input values, as these were exclusively matches with faint stars near the detection limit. We then computed, as a function of both $V$ magnitude and position in the frame, the completeness, the median shift in $V$ and $V-I$ experienced by the stars, and the standard deviation of $V_{\text {out }}-V_{\text {in }}$ and $(V-I)_{\text {out }}-(V-I)_{\text {in }}$ (using Tukey biweights to limit the effects of outliers), which we call $\sigma_{V}$ and $\sigma_{V-I}$.

\subsection{The LMC with $H S T$}

Olsen et al. (1998; hereafter O98) presented a $V-I, V$ color-magnitude diagram of NGC 1835 derived from HST WFPC2 observations. Figure 9 shows the F555W image of NGC 1835 and the accompanying $V-I, V$ CMDs within 4 annuli centered on the cluster. We used the results of artificial star simulations conducted in O98 to further test our crowding model. O98 added a set of $\sim 56000$ artificial stars with $26 \leq V \leq 16, \sim 500$ at a time, to copies of the original images of NGC 1835. The colors and magnitudes of these stars were chosen to mimic the observed CMDs; thus, their distribution resembles that of an LMC field star CMD. O98 performed photometry on the artificial images with DoPHOT (Schechter, Mateo, \& Saha 1993), and recovered the detected artificial stars by searching for matches between the input and output lists using a 0.6-pixel search radius. This approach of using the real images sprinkled with a few artificial stars at a time differs from the one described in section 3.1. However, both approaches should adequately measure the completeness and photometric errors due to crowding. 


\subsection{The LMC with the 30-m GSMT}

The design of the 30-m GSMT is currently under study by A.U.R.A.'s New Initiatives Office. A science case and "point design" have been developed, which are fully described in Web documents ${ }^{2}$. The point design features a primary mirror composed of $>600$ hexagonal segments producing a 30$\mathrm{m}$ filled aperture. A multi-conjugate adaptive optics (MCAO) system intends to provide near diffraction-limited resolution, with Strehl ratio of $\sim 0.5$, in the near-infrared $(J H K)$ over a $\sim 2^{\prime}$ field of view.

To generate an artificial GSMT image of NGC 1835, we adopted a hypthetical 4096 x 4096 near-infrared camera with 0 '!005 pixels and read noise of $15 e^{-}$per pixel. We estimated the system throughput by scaling an 8-m class telescope to 30-m, including all mirrors, the atmosphere, and an MCAO module. At $J$, the total system throughput is 0.31 ; at $K$, it is 0.40 . Using the list of stars produced for the seeing-limited simulation (section 3.1), we added the appropriate stellar flux to individual pixels in two $4096 \times 4096$ arrays representing the $J$ and $K$ images. The faintest of these stars have $J \sim 28$ corresponding to the lowest mass stars available in the Girardi et al. (2000) isochrones. We then convolved the images with simulated MCAO PSFs, the details of which are described at http://www.aura-nio.noao.edu/book/index.html. To describe them briefly, the PSFs have diffraction-limited cores with FWHM of 0.'009 in $J$ and 0!.015 in $K$. The Strehl ratios are 0.2 in $J$ and 0.6 in $K$. These PSFs include the effects introduced by the limited number of actuators in the deformable mirrors, the limited temporal sampling of the wavefront, spatial aliasing caused by the limited resolution of the wavefront sensors, and the estimated optical effects of the primary mirror segments (tilt and segment-to-segment dephasing). The PSFs are unrealistic in a few ways, however. First, we assume that anisoplanatism is completely taken out by the MCAO system, hence the PSFs do not vary with position in the image. The power of MCAO lies in its ability to produce a highly constant PSF across the field of view; but if the PSF changes by small amounts in the central $1^{\prime}$ of the field, it will introduce some small extra photometric error. Second, the PSFs do not vary with time, which in practice may be the limiting factor for the accuracy of the absolute photometric calibration. That is, if observations of uncrowded fields are needed to calculate aperture corrections for the crowded fields out to radii of several arcseconds, then time variability of the PSF will produce an impact. We consider our neglect of these two effects largely irrelevant to this study, the goal of which is mainly to determine the magnitude levels at which crowding is the dominant source of photometric error. Third, because of the large size $(2048 \times 2048$ pixels $)$ of the PSF arrays, we found it intractable to interpolate the full PSFs to higher spatial sampling. Thus, the stars in our simulated images all appear at the centers of the pixels. Following a suggestion from the referee, we tested the effect of this simplification by using a 200-pixel wide subsection of the GSMT PSFs, which we interpolated to sub-pixel positions before placing stars in the simulated images. These PSF subsections contain the cores and diffraction rings, as well as most of the halo structure, but neglect $\sim 10 \%$ of the total light. The photometric

\footnotetext{
${ }^{2}$ http://www . aura-nio.noao.edu/book/index.html
} 
errors recovered from these simulations were identical to those with stars placed only at pixel centers. We thus conclude that the simplification of placing stars only at pixel centers has no effect on the conclusions presented here.

After the PSF convolution, we produced the final simulated images assuming that the observation contained a set of 100 0.5-second exposures and 100 10-second exposures. These images include a sky background of $16.2 \mathrm{mag} \operatorname{arcsec}^{-2}$ in $J$ and $13.7 \mathrm{mag} \operatorname{arcsec}^{-2}$ in $K$, Poisson noise from the sky and astronomical sources, and a saturation cutoff of 65535 ADU assuming gain of 2 $e^{-} /$ADU. We did not simulate the 200 images directly; we instead simulated the average of the two sets of 100 images, which have photon noise and read noise reduced by a factor of 10 compared to the individual images. Following the procedures described in section 3.1, we performed photometry

on the images with DAOPHOT/ALLSTAR, including derivation of the PSFs but not including measurement of aperture corrections. We merged the short- and long-exposure photometry and determined the completeness and photometric errors from the recovered artificial stars. Figure 10 shows the $K$ image alongside the derived $J-K, K$ CMDs in four annuli centered on the cluster.

\subsection{Results}

Using equations 8 and 12, we computed the predicted crowding-induced photometric errors for the NGC 1835 fields of each telescope as a function of magnitude within 4-6 annuli centered on the cluster. These predictions require that we know the distance to NGC 1835 and its surrounding field, the age, metallicity, and surface brightness of the stellar population, and the imaging resolution of the telescope. For the seeing-limited and GSMT cases, we used the surface brightness profile, distance modulus, and luminosity function used to generate the input population. For the $H S T$ simulation, we used the observed surface brightness profile (O98, scaled to match the central surface brightness of Mateo 1987), a distance modulus of 18.5, and the luminosity function derived from the Girardi et al. (2000) isochrone of a 14 Gyr-old population with $[\mathrm{Fe} / \mathrm{H}]=-1.5$. For the seeinglimited and GSMT cases, we used the average FWHM of the profiles of stars scattered throughout the images to compute $a_{\text {res. }}$. For the $H S T$ case, we used two values of $a_{\text {res }}$ : one corresponding to the diffraction-limited resolution of the telescope ( 0 "'06 for F555W and $0{ }^{\prime \prime} 085$ for F814W) and another equal to the 2-pixel resolution of the Planetary Camera, 0"'1 in both F555W and F814W.

\subsubsection{Seeing-limited case}

Figure 11 shows the run of $\sigma_{V}$ and $\sigma_{V-I}$ with $V$ for the seeing-limited case, compared with our analytical predictions and with the photometric errors reported by DAOPHOT. While the errors reported by DAOPHOT grossly underestimate the true errors, our predictions of the dependence of $\sigma_{V}$ on $V$ agree within $\sim 20 \%$ with the simulation results over almost the entire range of surface brightnesses $\Sigma_{V}$. The good agreement between the simulated photometric errors and our crowding 
model clearly demonstrates that crowding is a dominant source of error in the seeing-limited LMC field. There are some disagreements, however. First, as a consequence of our assumption of a tophat PSF and the hard limits of integration in equation 8, the appearance of the horizontal branch produces a discontinuity in the analytical $\sigma_{V}$ at $V \sim 19$, which is not seen in the simulations. Second, at high surface brightnesses $\left(\Sigma_{V}<17.6\right)$, the simulation predicts much smaller photometric errors than does the analytical model. We note, however, that at these surface brightnesses there is a systematic bias towards recovering stars brighter than their input magnitudes. Clearly, these input stars are only recovered if they sit on top of large background fluctuations that push them above the detection limit, as seen by the poor fit of the input isochrone to the CMDs. Although this bias causes stars to be recovered with magnitudes quite different from their input values, the dispersion $\sigma_{V}$ in the recovered magnitudes actually decreases, because stars that would have been recovered fainter than their input magnitudes simply aren't detected. In other words, we are able to sample only a narrow tail of the photometric error distribution through the simulations, making our predictions are invalid. This bias could also explain the effect seen at faint magnitudes for all surface brightnesses, where taken at face value the simulation results suggest decreasing photometric errors with increasing magnitude. The low completeness at these magnitudes insures that only the tail of the photometric error distribution is observed.

Our predictions of the run of $\sigma_{V-I}$ with $V$ are also in reasonable agreement with the simulation. Both the simulation and our analytical predictions show that $\sigma_{V-I}$ is lower than $\sigma_{V}$ at fixed $V$, which we attribute to the correlated crowding-induced photometric errors in $V$ and $I$. Again, the DAOPHOT errors tend to underestimate the true errors.

\subsubsection{HST case}

Figure 12 shows $\sigma_{V}, \sigma_{V-I}$, and the CMDs for six annuli centered on NGC 1835 as derived from the HST photometry. As in the seeing-limited case, the errors reported by DoPHOT grossly underestimate the true $\sigma_{V}$. Our predicted $\sigma_{V}$ values, on the other hand, again generally agree with the simulation results to within $\sim 20 \%$. The two resolutions (diffraction-limited resolution, 0 ' $06^{\prime}$ in $V$ and $0{ }^{\prime} .085$ in $I$, and 2-pixel, $0{ }^{\prime} 1$ PC resolution) used to calculate the predictions bracket the simulation results, demonstrating that WFPC2's undersampling of the PSF affects the crowdinginduced photometric errors. Undersampling likely also explains why the DoPHOT photometry never achieves an accuracy higher than $\sim 5 \%$ at bright magnitudes, where our crowding model predicts that better performance should be possible; similar results have been found by others (e.g. Dolphin 2000).

As for the seeing-limited case, our predictions of $\sigma_{V-I}$ for the $H S T$ case agree with the simulations in producing colors that are more accurately measured than magnitudes. However, the errors in color predicted by DoPHOT agree better with the simulation results than do our predictions, which include only the effects of crowding. Thus, for the HST case, we conclude that sources other than crowding dominate the color error budget. 


\subsubsection{GSMT case}

Figure 13 shows $\sigma_{J}, \sigma_{J-K}$, and the $J-K, J$ CMDs for the GSMT LMC cluster simulation. Adopting $\lambda / D$ as the diffraction-limited resolution, i.e. 0.'009 in $J$ and $0{ }^{\prime \prime} 015$ in $K$, our analytical predictions suggest that crowding dominates the errors in both magnitude and color. As for the other simulations, the errors reported by DAOPHOT tend to underestimate the true errors.

We note that the high photometric accuracies $(\lesssim 1 \%)$ that we achieve with our GSMT photometry for the bright, uncrowded stars are unrealistic. For these stars, we expect that errors in the PSF model should dominate the error budget. Esslinger \& Edmunds (1998), based on both simulations and observations with the ADONIS AO system on the ESO 3.6-m telescope, found typical random photometric errors of $\sim 0.05$ magnitudes for uncrowded stars. While MCAO should significantly improve the minimum achievable photometric error, we adopt $5 \%$ minimum photometric errors in the worst-case scenario. For most applications, such an error will not significantly affect the ability to measure the ages and metallicities of stellar populations.

\subsubsection{Completeness}

Figure 14 demonstrates the intimate connection between photometric error and completeness, as determined from the simulations of all three telescopes (seeing-limited, $H S T$, and GSMT). For

the seeing-limited case, we have excluded the regions with $\Sigma_{V} \leq 17.6$ where the simulation becomes biased towards lower photometric errors, as discussed above. We expect that completeness should depend on the details of the object detection algorithm and the fit of the PSF to the objects. Indeed, we see slightly different shapes to the curves in Figure 14 for the three sets of simulations. However, Figure 14 demonstrates that in observations of crowded fields with sufficient exposure time to render photon noise negligible, completeness is dominated by the difficulty of detecting and measuring objects against the fluctuating background, independent of the shape of the PSF and the details of the analysis. From Figure 14, we arrive at a useful rule of thumb: completeness drops sharply when photometric errors due to crowding exceed $\sim 0.1$ magnitudes.

In summary, our simple analytical model of crowding is able to predict the magnitude error due to crowding in any environment and observed with any resolution to an accuracy of $\sim 20 \%$. It is also able to reproduce the observation that colors are measured more accurately than expected, although the calculation of color error is fairly sensitive to the input parameters. Our model demonstrated that our GSMT simulations realistically reproduce the effects of crowding, in spite of the idealizations made.

In the next sections, we compare our crowding model to photometry measured with a real AO system. We also explore further what ELTs, such as the GSMT, can do in the study of resolved stellar populations. 


\section{M32 with Gemini North + Hokupa'a, NGST, and GSMT}

\subsection{Photometric errors in M32 with Gemini + Hokupa'a}

M32 is a common benchmark for population synthesis studies of more distant elliptical galaxies, and thus will be an important target for ELTs. Integrated spectroscopy combined with population synthesis analyses conclude that the inner region of M32 is intermediate $(\sim 4-5 \mathrm{Gyr})$ in age and has solar metallicity (Trager et al. 2000, del Burgo et al. 2001). Davidge et al. (2000; hereafter D00) observed the central 20" of M32 with Gemini North and Hokupa'a, and detected AGB stars to $M_{K} \sim-5$. While the $\sim 00^{\prime \prime} 12$ resolution of their images was insufficient to test whether the population synthesis models are correct, they established that there are no radial trends in the luminous AGB population within the inner $20^{\prime \prime}$.

Figure 2 of D00 shows a decrease in completeness and increase in photometric error at fixed magnitude with rising surface brightness, demonstrating that crowding is a dominant source of photometric error in the Hokupa'a M32 photometry. Moreover, the inner 2" contains almost purely blended stars, such that crowding prohibits any useful photometry.

How does the photometric error due to crowding compare with the other sources of photometric error, such as imperfections in the PSF fits? To answer this question, we performed artificial star tests using D00's M32 images. First, we followed the prescription for performing photometry outlined by D00 and references therein to demonstrate that we could reproduce the D00 CMDs. In brief, we removed the variable unresolved background by median-filtering the $H$ and $K$ images through a 100 pixel wide filter and subtracting these from the originals. We then performed photometry with DAOPHOT/ALLSTAR, as done in Section 3. However, we did not compute our own aperture corrections; instead, we applied offsets to the $H$ and $K$ magnitudes to bring them to agreement with the D00 photometry, which Tim Davidge kindly provided to us. Next, we added 14500 artificial stars drawn from a mix of $1 \mathrm{Gyr}([\mathrm{Fe} / \mathrm{H}]=0), 5 \mathrm{Gyr}([\mathrm{Fe} / \mathrm{H}]=0)$, and $10 \mathrm{Gyr}([\mathrm{Fe} / \mathrm{H}]$ $=-0.3$ ) populations to copies of the original images. The 1, 5, and $10 \mathrm{Gyr}$ old stars were assumed to comprise $10 \%, 45 \%$, and $45 \%$ of the total by mass, respectively, roughly following the estimated age distribution of Grillmair et al. (1996); we adopted a Salpeter IMF for these populations. We added the stars to the $H$ and $K$ images 250 at a time, following a spatial distribution mimicking that of M32 itself, and performed photometry on the modified images exactly as done for the originals. We identified the stars recovered in the simulation by matching the output star list to the input star list, using a 1-pixel search radius.

Figure 15 shows the Gemini+Hokupa'a M32 CMD that we derive within the annulus between $7{ }^{\prime \prime} .4$ and $133^{\prime \prime} 1$ radius centered on M32. We have excluded stars with $y$ pixel positions greater than 700 , because in this region we found the effects of anisoplanatism on the PSF to be severe and difficult to model. Figure 15 also shows the photometric errors $\sigma_{H}, \sigma_{K}$, and $\sigma_{H-K}$ derived from the artificial star tests, along with our analytical predictions. The predictions assume the same

mix of stellar populations used in the artificial star tests, a distance modulus of 24.3 for M32, and 
0 '. $12 / 0$ '. 14 resolution in $H / K$ (D00). We find that the photometric errors are consistent with being entirely due to crowding down to a level of $\sim 5 \%$. We thus claim that other sources of random photometric error must be $\lesssim 5 \%$. This test demonstrates that despite the low Strehl of its PSFs, Gemini+Hokupa'a photometry in crowded fields is limited by the resolution of the PSF core.

\subsection{Simulated NGST performance}

The Next Generation Space Telescope (NGST) aims to provide diffraction-limited performance in the near-infrared with an $\sim 8$-m aperture. Because of the low background in space and the $\sim 100 \%$ Strehl ratio of the PSF, the NGST will provide huge gains in sensitivity over AO-corrected groundbased telescopes of similar size. However, for crowding-limited photometry, where the limiting factor is the size of the diffraction-limited core of the PSF, the NGST will provide no such gain. To demonstrate this, we simulated an NGST observation of a field in M32 covering the region observed by D00. To generate the input star list, we assumed the same populations used in the Gemini+Hokupa'a artificial star tests, i.e. $10 \% 1 \mathrm{Gyr}([\mathrm{Fe} / \mathrm{H}]=0), 45 \% 5 \mathrm{Gyr}([\mathrm{Fe} / \mathrm{H}]=0)$, and $45 \% 10$ Gyr $([\mathrm{Fe} / \mathrm{H}]=-0.3)$ stars. The faintest stars in the simulation have $K \gtrsim 27$, well below the expected completeness limit set by crowding. We added these stars to $586 \times 586$ arrays according to the spatial profile of Kent (1987), assuming 0".035 pixels, which is appropriate for the proposed NIRCam ${ }^{3}$. As we did with the GSMT simulations, we added all of the stars to the centers of pixels. We then convolved these arrays with Krist's (1999) $J$ and $K$ PSFs, which were calculated for an 8-m diameter NGST. These PSFs have diffraction-limited cores of $0 . \prime 032$ at $J$ and 0.057 at $K$; as such, our pixel scale results in severe undersampling of the $J$ PSF. We interpolated these PSFs to the 0. '035 pixel scale before convolution.

We performed photometry on the simulated images and analyzed the output star list following the procedures outlined in Section 3. Figure 16 shows the CMD in the 7". $4-13^{\prime \prime} .1$ annulus centered on M32 compared with the input isochrones. The CMD is deeper than that of Gemini+Hokupa'a, as is to be expected from the higher resolution of NGST. The photometric errors appear consistent with being dominated by crowding, as seen by the comparison of the simulation results with our analytical predictions in Figure 16. These predictions adopt as input the properties of the artificial populations; we use the diffraction-limited resolution for $K$ but two resolutions for $J, 0$ '.035 and 0 . 070 . The $J$ photometry is clearly affected by the undersampling, as the simulation results fall closer to the 2-pixel resolution curve than the diffraction-limited resolution curve.

\footnotetext{
${ }^{3}$ http://www.stsci.edu/ngst/instruments/nircam/
} 


\subsection{Simulated GSMT performance}

Because of the effects of crowding, previous studies of M32 leave the nature of M32's underlying stellar population highly uncertain. With its much higher resolution, the GSMT can make a large impact on our understanding of this galaxy. To study the GSMT's performance further, we simulated a GSMT observation of the center of M32.

We generated simulated $J$ and $K$ images of M32 using the same input star list and spatial distribution as for the NGST simulation. We added the stars to $4096 \times 4096$ arrays, convolved the arrays with our simulated GSMT PSFs, and scaled the resulting $J$ and $K$ images to exposure times of $5 \times 10$ s and $15 \times 120$ s using the system efficiencies from Section 3.3. We analyzed these images with DAOPHOT, as was done for the LMC GSMT simulation.

Figure 17 shows the simulated $J-K, K$ CMD in the $7.44-13^{\prime \prime} 1$ annulus centered on M32, which may be compared to Figs. 15 and 16, and the photometric error profiles $\sigma_{J}, \sigma_{K}$, and $\sigma_{J-K}$. The depth of the photometry $(J \sim 26.5)$ is comparable to the faintest magnitude of the input population $(J \sim 27.6)$. However, because there is reasonable agreement of the analytical model with the simulation and we understand that the effects of crowding are dominated by fluctuations produced by stars with similar brightnesses to the star of interest, we conclude that the input star list is sufficiently deep to adequately model the effects of crowding. The simulation shows that near the center of M32, the GSMT will detect stars with $M_{J} \sim 0.5$, below the turnoff of a 1 Gyr population. Farther from the dense center, such as in the region studied by Grillmair et al. (1996), the GSMT will also detect the older populations. If a 5 Gyr-old population is present, as suggested by the population synthesis models, then a telescope such as the GSMT will be able to observe it. Compared to the NGST, the GSMT will reach depths $\sim 3$ magnitudes deeper.

\section{Beyond the Local Group: 30-m vs. 100-m, IR vs. optical}

An important goal for stellar populations research is to measure the ages, abundances, and kinematics of stars in a statistically significant sample of galaxies covering a range of morphologies and environments, so as to overcome the limitations of cosmic variance (Wyse et al. 2000). How

will the performance of a 30-m telescope compare to that of a 100-m telescope? The high surface brightnesses of most galaxies means that crowding will dominate the photometric errors; equation 8 may thus be used to assess the relative performances of different apertures and operating wavelengths.

Using equation 8, we calculated crowding limits for 30-m and 100-m apertures assuming diffraction-limited resolution in $V$ and $K$. We assumed a stellar surface brightness of $\Sigma_{V}=$ $22, \Sigma_{K}=19$ and a 14 Gyr-old population with $[\mathrm{Fe} / \mathrm{H}]=-1$. As discussed in Section 2, using other metallicities and ages could alter the results considerably. Figures 18 and 19 show the limits at which $\sigma=0.1$ photometry may be performed with $30-\mathrm{m}$ and $100-\mathrm{m}$ telescopes, respectively, at 
the distances of the LMC, M31, the Sculptor group, Cen A, and the Virgo cluster. Our limits show that near the half-light radii of giant elliptical galaxies in the Virgo cluster, the 30-m GSMT will produce results similar to what is now possible in the core of M32. CMDs could be produced down to the base of the giant branch in regions of the M31, bulge and disk, down to the horizontal branch in the disks of Sculptor group galaxies such as NGC 253, and to below the tip of the red giant branch in Cen A. To reach the old main sequence turnoff in a galaxy as distant as M31, the GSMT will be restricted to working in regions of surface brightness $\Sigma_{K} \gtrsim 21$. If diffraction-limited resolution were possible at wavelengths as short as $5000 \AA$, then the age and metallicity distribution of the M31 bulge and disk could be measured, the star formation histories of Sculptor group galaxies would be accessible to study, and the horizontal branch detected in Cen A. In the Virgo cluster, we could observe the TRGB to almost the half-light radii of giant ellipticals.

A 100-m telescope, such as the Overwhelmingly Large telescope (OWL) being studied by the European Southern Observatory, operating in the near-infrared will accomplish roughly what a diffraction-limited 30-m telescope can do in the optical. With diffraction-limited resolution in the optical, the 100-m could measure the complete star formation histories and abundance distributions in galaxies out to the distance of Cen $\mathrm{A}$; it could even reach the old main sequence turnoff in low surface brightness regions $\left(\Sigma_{V} \sim 25\right)$ of Virgo cluster galaxies.

\section{Summary and Conclusions}

In this paper, we have developed and used an analytical model to predict the magnitude errors produced by crowding with an accuracy of $\sim 20 \%$. We have tested our predictions with Monte Carlo simulations of astrophysical environments covering several magnitudes of surface brightness, as observed with telescopes spanning two orders of magnitude in resolution. We have shown that correlations between the crowding-induced errors in different photometric bands leads to significantly smaller errors in color than would otherwise be expected. Using both Monte Carlo simulations and our analytical predictions, we have shown to what level 30-m and 100-m AO-corrected telescopes will perform useful photometry for galaxies at distances as large as the Virgo cluster.

Throughout, we have neglected the amount of integration time needed to reach the desired magnitude. How long will it take for a given ELT to reach its crowding limit? From equation 8, we see that the magnitude limit of a telescope for crowding-limited stellar photometry is proportional to the area of a resolution element. For a seeing-limited telescope, we reach the obvious conclusion that building a bigger telescope does not improve the crowding limit; however, the time needed to reach the crowding limit decreases proportionally to the area of the aperture. On the other hand, if the telescope is diffraction-limited, as is expected to be the case for AO-corrected ELTs, the depth of the crowding limit scales roughly with the collecting area of the telescope, neglecting the effect of the detailed shape of the luminosity function. Since the flux received also scales with the collecting area, this means that the time it takes for a diffraction-limited telescope to reach its confusion limit is independent of aperture size. As an example, from section 3.4 we found that WFPC2 with HST 
is limited to $\sigma_{V}=0.1$ at $V \sim 23.5$ when the stellar background contributes $21.0 \mathrm{mag} \operatorname{arcsec}^{-2}$, which is the typical surface brightness in spiral arms (e.g. Okamura 1988) and at the effective radius of giant elliptical galaxies (e.g. Caon et al. 1990). According to the WFPC2 exposure time calculator, the exposure time needed to achieve $\mathrm{S} / \mathrm{N}=10$ at $\mathrm{V}=23.5$ against a background of 21.0 mag $\operatorname{arcsec}^{-2}$ is $\sim 90$ seconds. Thus, a diffraction-limited telescope is rapidly limited by crowding in regions with surface brightnesses typical of normal galaxies. Even in the infrared observed from the ground, a GSMT with Strehl of 1.0 would reach its crowding limit in $<300$ seconds when observing a field with $\Sigma_{K}=18 \mathrm{mag} \operatorname{arcsec}^{-2}{ }^{4}$. The rapid approach of the crowding limit argues that the Strehl ratios of ELT PSFs need not be large for crowded-field photometry. The huge difference in spatial scale between the diffraction-limited core and the seeing-limited "halo" for ELTs means that lower Strehl simply raises the sky background and increases the exposure time needed to reach the crowding limit. As seen here, diffraction-limited telescopes operating in crowded environments have plenty of exposure time to spare.

On a more general note, artificial star tests are useful and necessary for many areas of stellar populations research, e.g. measuring LFs in clusters and determining the star formation histories of galaxies from field CMDs. However, as demonstrated here and elsewhere, care must still be taken to ensure that the simulations faithfully represent reality. The tools developed here may provide a sanity check on the photometric errors and completeness levels determined from simulations. For some applications, one may even be able to replace the artificial star tests with our analytical crowding estimates.

This work grew out of discussions held at the NOAO GSMT stellar populations panel, which was chaired by Rosie Wyse. We are grateful for her leadership of this panel and for follow-up discussions with her afterwards. We also thank Jay Frogel, Mario Mateo, Joan Najita, Andrew Stephens, and Steve Strom for their input and wisdom. We thank Tim Davidge for kindly providing us with his M32 photometry. We are indebted to the anonymous referee, who provided a thorough and thought-provoking report which substantially improved this paper. Finally, KO thanks Dara Norman for continued inspiration.

\footnotetext{
${ }^{4}$ calculated using IRAF CCDTIME, 30-m primary with 1.5-m central obscuration, and MCAO imager as described in text
} 


\section{REFERENCES}

Binney, J., \& Merrifield, M., 1998, Galactic Astronomy (Princeton: Princeton University Press), p. 124

Caon, N., Capaccioli, M., Rampazzo, R. 1990, A\&AS, 86, 429

Condon, J. J. 1974, ApJ, 188, 279

Davidge, T., Rigaut, F., Chun, M., Brandner, W., Potter, D., Northcott, M., Graves, J. E. 2000, ApJ, 545, 89

del Burgo, C., Peletier, R. F., Vazdekis, A., Arribas, S., Mediavilla, E. 2001, MNRAS, 321, 227

Dolphin, A. E. 2000, PASP, 112, 1383

Esslinger, O., \& Edmunds, M. G. 1998, A\&AS, 129, 617

Hogg, D. W. 2001, AJ, 121, 1207

Gallart, C, Aparicio, A.,\& Vílchez, J. M. 1996, AJ, 112, 1928

Girardi, L., Bressan, A., Bertelli, G., \& Chiosi, C. 2000, A\&AS, 141, 371

Grillmair, C. J., Lauer, T. R., Worthey, G., Faber, S. M., Freedman, W. L., Madore, B. F., Ajhar, E. A., Baum, W. A., Holtzman, J. A., Lynds, C. R., O’Neil, E. J., Jr., Stetson, P. B. 1996, AJ, 122, 1975

Kent, S. M. 1987, AJ, 94, 306

King, I. R. 1966, AJ, 71, 64

Landolt, A. U. 1992, AJ, 104, 340

Mateo, M. 1987, ApJ, 323, L41

Okamura, S. 1988, PASP, 100, 524

Olsen, K. A. G., Hodge, P. W., Mateo, M., Olszewski, E. W., Schommer, R. A., Suntzeff, N. B., \& Walker, A. R. 1998, MNRAS, 300, 665

Olsen, K. A. G. 1999, AJ, 117, 2244

Renzini, A. 1998, AJ, 115, 2459

Salpeter, E. E. 1955, ApJ, 121, 161

Schechter, P. L., Mateo, M., \& Saha, A. 1993, PASP, 105, 1342

Scheuer, P. A. G. 1957, Proc. Cambridge Philos. Soc., 53, 764 
Stephens, A. W., Frogel, J. A., Freedman, W., Gallart, C., Jablonka, P., Ortolani, S., Renzini, A., Rich, R. M., Davies, R. 2001, AJ, 121, 2584

Stetson , P. B. 1987, PASP, 93, 1439

Strom, S. et al. 2002, "Enabling a Giant Segmented Mirror Telescope for the Astronomical Community," http://www .aura-nio.noao.edu/book/index.html

Tonry, J. \& Schneider, D. P. 1988, AJ, 96, 807

Trager, S. C., Faber, S. M., Worthey, G., Gonzlez, J. J. 2000, AJ, 119, 1645

Wyse, R. F. G. et al. 2000, "Report of the Stellar Populations Panel," http://www . aura-nio.noao.edu/book/ch2/2_C.pdf 
Fig. 1.- Left: $V$-band luminosity functions for solar metallicity stellar populations from the Girardi et al. (2000) models, assuming a Salpeter IMF. Each luminosity function is labelled with the assumed age, which runs from 1 to 10 Gyr. The curves have been multiplied by arbitrary scale factors for display purposes. Right: The surface brightnesses at which crowding limits photometry to $10 \%$ accuracy, displayed as a function of age for stars with $11 \leq M_{V} \leq-3$. The calculation assumes spatial resolution of $1^{\prime \prime}$ and a distance modulus of 18.5. To translate the predictions to other resolutions $a_{\text {res }}$ or distances $(m-M)_{0}$, one simply needs to add constants of $2.5 \log \left(a_{\text {res }}^{2}\right)$ and $(m-M)_{0}-18.5$ to the values on the $y$ axis.

Fig. 2.- Left: $V$-band luminosity functions for 5 Gyr stellar populations from the Girardi et al. (2000) models, assuming a Salpeter IMF. Each luminosity function is labelled with the assumed metallicity. The curves have been multiplied by arbitrary scale factors for display purposes. Right: The surface brightnesses at which crowding limits photometry to $10 \%$ accuracy, displayed as a function of metallicity for stars with $11 \leq M_{V} \leq-3$. As in Fig. 1, the calculation assumes spatial resolution of $1^{\prime \prime}$ and a distance modulus of 18.5 .

Fig. 3.- Left: $V$-band luminosity functions for $5 \mathrm{Gyr}$ solar metallicity stellar populations from the Girardi et al. (2000) models. Each luminosity function is labelled with the assumed slope of the IMF, where 2.35 corresponds to a Salpeter IMF. The curves have been multiplied by arbitrary scale factors for display purposes. Right: The surface brightnesses at which crowding limits photometry to $10 \%$ accuracy, displayed as a function of IMF slope for stars with $11 \leq M_{V} \leq-3$. As in Fig. 1, the calculation assumes spatial resolution of $1^{\prime \prime}$ and a distance modulus of 18.5. 
Fig. 4.- Left: $K$-band luminosity functions for solar metallicity stellar populations from the Girardi et al. (2000) models, assuming a Salpeter IMF. Right: The surface brightnesses at which crowding limits photometry to $10 \%$ accuracy, displayed as a function of age for stars with $7 \leq$ $M_{K} \leq-5$. See Fig. 1 caption for details.

Fig. 5. - Left: $K$-band luminosity functions for 5 Gyr stellar populations from the Girardi et al. (2000) models, assuming a Salpeter IMF. Right: The surface brightnesses at which crowding limits photometry to $10 \%$ accuracy, displayed as a function of metallicity for stars with $7 \leq M_{K} \leq-5$. See Fig. 1 caption for details.

Fig. 6.- Left: $K$-band luminosity functions for 5 Gyr solar metallicity stellar populations from the Girardi et al. (2000) models. Right: The surface brightnesses at which crowding limits photometry to $10 \%$ accuracy, displayed as a function of IMF slope for stars with $7 \leq M_{K} \leq-5$. See Fig. 1 caption for details.

Fig. 7. - NGC 1835 from the ground. Left: This $V$ image was taken by A. Walker in January 1995 with the CTIO 1.5-m telescope and Tek $2048 \times 2048$ camera. The resolution is $\sim 1^{\prime \prime}$ and the field of view is $\sim 8^{\prime} \times 8^{\prime}$. Right: $V-I, V$ color-magnitude diagram from the CTIO 1.5-m image displayed on the left, shown in four annuli centered on NGC 1835; the limits of the radial bins are labeled in the plots. The inner annuli contain predominantly cluster stars, while the outer annuli are dominated by the LMC field.

Fig. 8.- Left: a simulated seeing-limited image of NGC 1835. Right: $V-I, V$ CMD derived from the simulated image shown on the left, displayed in the same four annuli centered on the cluster shown in Fig. 7. 
Fig. 9. - Left: HST WFPC2 F555W image of NGC 1835, from O98. The resolution is $\sim 0$ ' 1 and the field of view is $\sim 2.5 \times 2 ! 5$. Right: $V-I, V$ CMD derived by O98 from the HST image, displayed in four annuli centered on NGC 1835, as labeled in the plots.

Fig. 10. - Left: Simulated $K$ image of NGC 1835 with the 30-m Giant Segmented Mirror Telescope. The resolution is $\sim 00^{\prime} 015$ and the field of view is $20^{\prime \prime} \times 20^{\prime \prime}$. Right: $J-K, J$ CMD derived from the simulated image, displayed in four annuli centered on the cluster.

Fig. 11. - Crowding in NGC 1835 from the ground. The middle column of plots shows the CMDs derived at various annuli in one of the simulated seeing-limited images of NGC 1835, compared with the input isochrone. The local surface brightnesses are labeled. In the left-hand column, the points indicate the photometric color errors $\sigma_{V-I}$ as derived from the simulation. The thick solid lines show our theoretical predictions of $\sigma_{V-I}$, the thin lines the errors reported by DAOPHOT. In the right-hand column, $\sigma_{V}$ is plotted instead.

Fig. 11.- cont.

Fig. 12.- Crowding in NGC 1835 with WFPC2 onboard HST. As in Fig. 11, the middle column of plots shows the CMDs derived from the observed images in various annuli centered on NGC 1835, compared with a representative $14 \mathrm{Gyr},[\mathrm{Fe} / \mathrm{H}]=-1.5$ Girardi et al. (2000) isochrone. The local surface brightnesses are labeled. In the left-hand column, the points indicate the photometric color errors $\sigma_{V-I}$ as derived from the simulation. The thick solid lines show our theoretical predictions of $\sigma_{V-I}$, the thin lines the errors reported by DAOPHOT. Predictions for both diffraction-limited (red lines) and 2-pixel resolutions (black lines) are shown; these predictions bracket the simulation results, demonstrating the effect of undersampling. In the right-hand column, $\sigma_{V}$ is plotted instead.

Fig. 12.- cont. 
Fig. 13. - Crowding in the simulated GSMT image of NGC 1835. As in Fig. 11, the middle column of plots shows the CMDs derived in various annuli centered on the cluster, compared with the input isochrone (red). The local surface brightnesses are labeled. In the left-hand column, the points indicate the photometric color errors $\sigma_{J-K}$ as derived from the simulation. The solid black lines show our theoretical predictions of $\sigma_{J-K}$ assuming diffraction-limited resolution (0!.009 in $J, 0^{\prime \prime} 015$ in $K$ ). The thin black lines show the errors reported by DAOPHOT. In the right-hand column, $\sigma_{J}$ is plotted instead.

Fig. 13.- cont.

Fig. 14.- Completeness in our NGC 1835 simulations is plotted against $\sigma_{V}$ (left) and $\sigma_{V-I}$ (right). Solid points show values from the seeing-limited simulation, open circles those from the $H S T$ simulation, and open triangles those from the GSMT simulation.

Fig. 15. - Gemini+Hokupa'a measurements of M32. At top left, the CMD derived for the annulus $7^{\prime \prime} .4 \leq r \leq 13$.' 1 centered on M32 in the Davidge et al. (2000) images is shown. The remaining three panels show the photometric errors $\sigma_{H-K}, \sigma_{H}$, and $\sigma_{K}$ derived from artificial star tests (filled circles) compared with our theoretical predictions. The predictions (thick lines) are for resolutions of $0^{\prime \prime} 12$ in $H$ and $0 ! 14$ in $K$, while the thin lines show the DAOPHOT-reported errors. 
Fig. 16. - Simulated M32 measurements with NGST. At top left the CMD derived for the annulus $7^{\prime \prime} .4 \leq r \leq 13$ '. 1 centered on M32 is shown compared with the input isochrones (red lines in electronic version, gray lines in print version). The remaining three panels show the photometric errors $\sigma_{J-K}$, $\sigma_{J}$, and $\sigma_{K}$ derived from the simulations (filled circles) compared with our theoretical predictions. The black lines show the predictions for diffraction-limited resolution in $J$ and $K\left(0{ }^{\prime \prime} 032\right.$ and $0{ }^{\prime \prime} 057$ respectively); the red lines (electronic version; dark gray lines in print version) show the predictions for 2-pixel ( 0 '. 7$)$ resolution in $J$. The green lines (electronic version; light gray lines in print version) show the DAOPHOT errors.

Fig. 17.- Simulated M32 measurements with GSMT. At top left the CMD derived for the annulus $7^{\prime \prime} 4 \leq r \leq 13$ "' 1 centered on M32 is shown compared with the input isochrones (red lines in electronic verision, gray lines in print version). The remaining three panels show the photometric errors $\sigma_{J-K}, \sigma_{J}$, and $\sigma_{K}$ derived from the simulations (filled circles) compared with our theoretical predictions. The black lines show the predictions for diffraction-limited resolution in $J$ and $K$ (0.'009 and 0.'015 respectively); the green lines (electronic version; light gray lines in print version) show the DAOPHOT errors.

Fig. 18. - Crowding limits with a 30-m AO-corrected telescope. The lines indicate the magnitudes at which $\sigma \lesssim 0.1$ photometry is possible in regions of surface brightness $\Sigma_{V}=22, \Sigma_{K}=19$ for galaxies at the indicated distances.

Fig. 19.- Crowding limits with a 100-m AO-corrected telescope. See Figure 18 for explanation. 


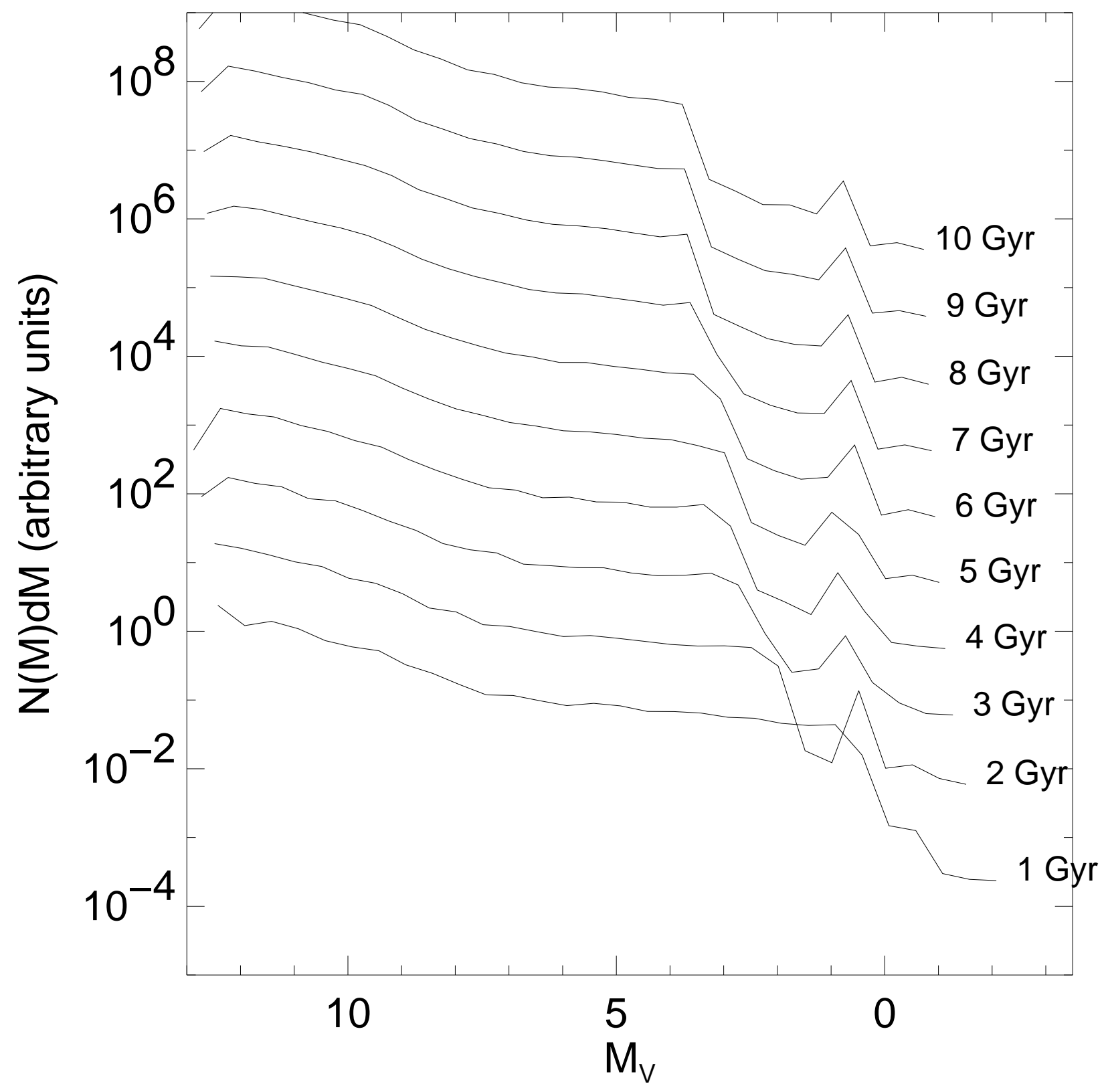


This figure "figure1b.png" is available in "png" format from: http://arxiv.org/ps/astro-ph/0304163v1 


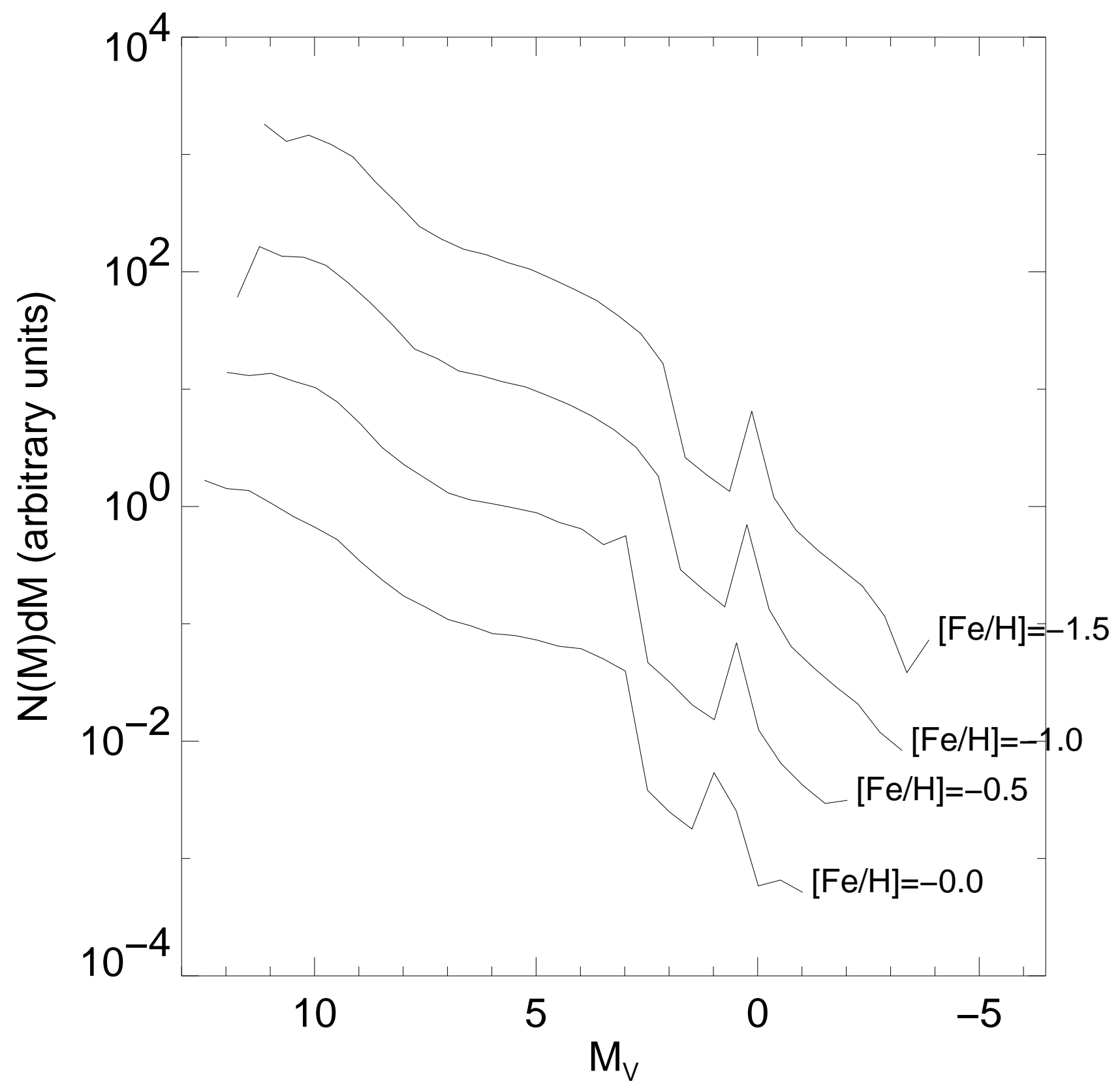


This figure "figure2b.png" is available in "png" format from: http://arxiv.org/ps/astro-ph/0304163v1 


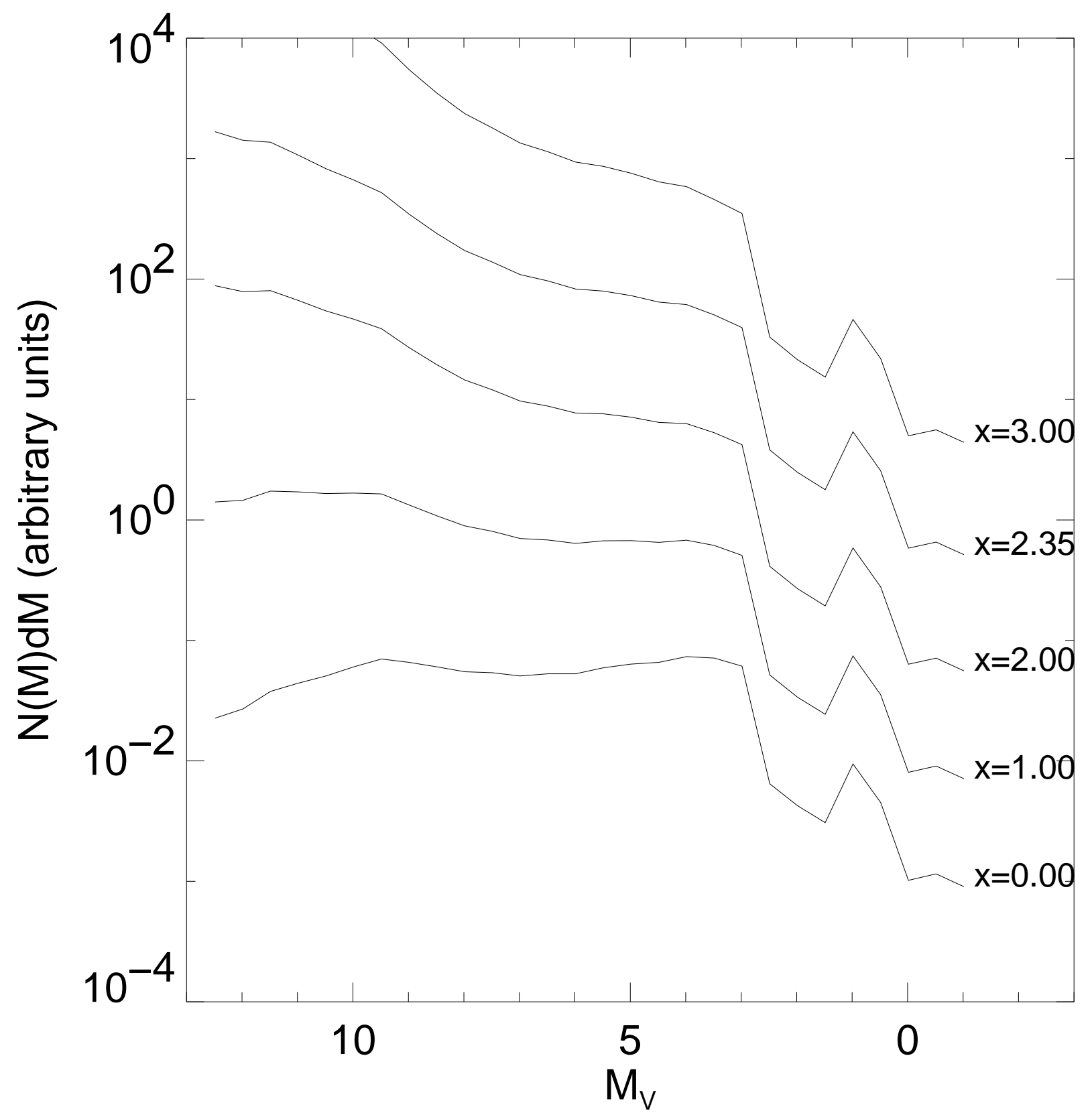


This figure "figure3b.png" is available in "png" format from: http://arxiv.org/ps/astro-ph/0304163v1 


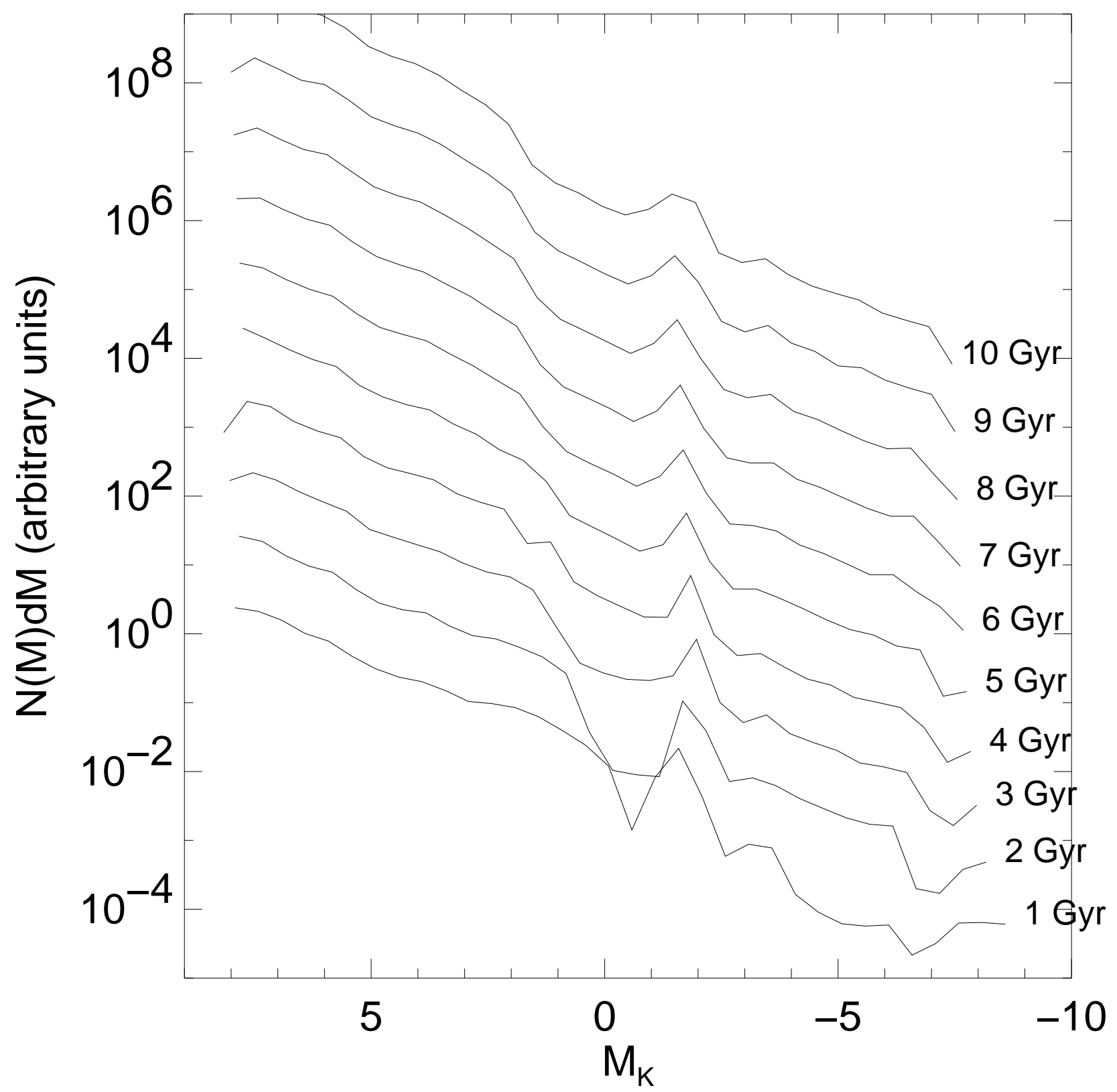


This figure "figure4b.png" is available in "png" format from: http://arxiv.org/ps/astro-ph/0304163v1 


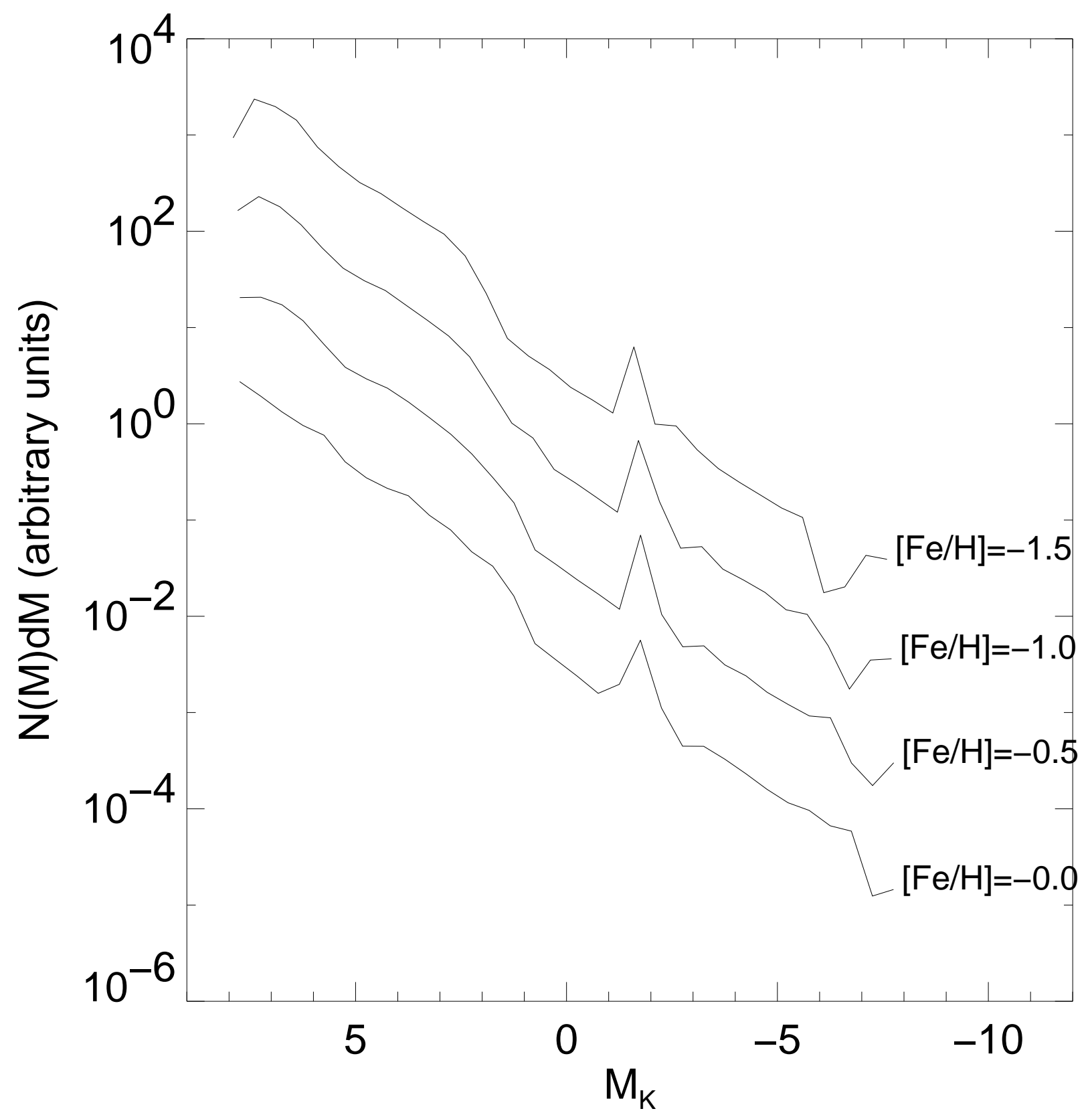


This figure "figure5b.png" is available in "png" format from: http://arxiv.org/ps/astro-ph/0304163v1 


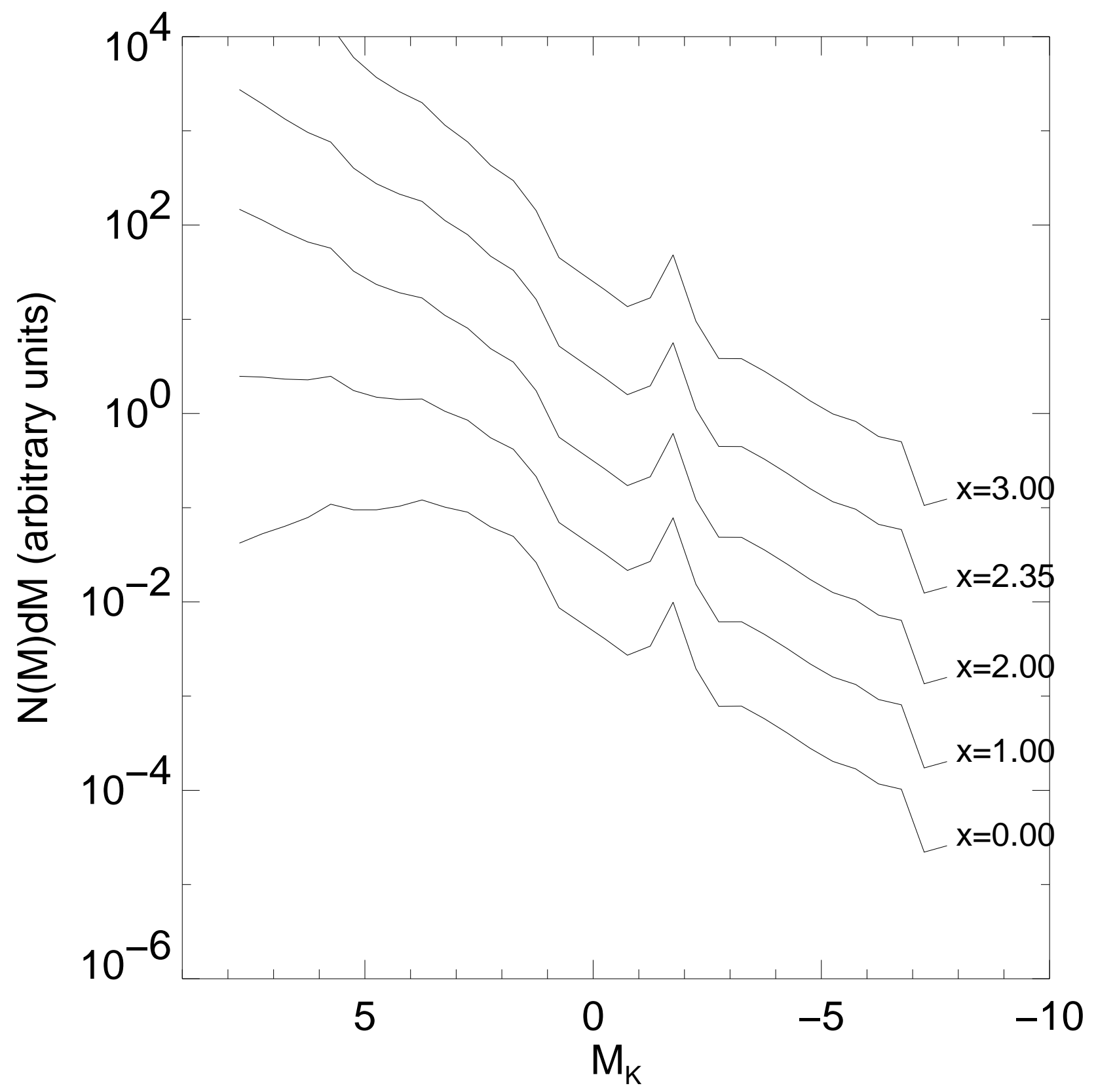


This figure "figure6b.png" is available in "png" format from: http://arxiv.org/ps/astro-ph/0304163v1 
This figure "figure7a.jpg" is available in "jpg" format from: http://arxiv.org/ps/astro-ph/0304163v1 
This figure "figure7a.png" is available in "png" format from: http://arxiv.org/ps/astro-ph/0304163v1 
This figure "figure7b.png" is available in "png" format from: http://arxiv.org/ps/astro-ph/0304163v1 
This figure "figure8a.png" is available in "png" format from: http://arxiv.org/ps/astro-ph/0304163v1 
This figure "figure8b.png" is available in "png" format from: http://arxiv.org/ps/astro-ph/0304163v1 
This figure "figure9a.png" is available in "png" format from: http://arxiv.org/ps/astro-ph/0304163v1 
This figure "figure9b.png" is available in "png" format from: http://arxiv.org/ps/astro-ph/0304163v1 
This figure "figure10a.png" is available in "png" format from: http://arxiv.org/ps/astro-ph/0304163v1 
This figure "figure10b.png" is available in "png" format from: http://arxiv.org/ps/astro-ph/0304163v1 
This figure "figure11a.png" is available in "png" format from: http://arxiv.org/ps/astro-ph/0304163v1 
This figure "figure11b.png" is available in "png" format from: http://arxiv.org/ps/astro-ph/0304163v1 
This figure "figure12a.png" is available in "png" format from: http://arxiv.org/ps/astro-ph/0304163v1 
This figure "figure12b.png" is available in "png" format from: http://arxiv.org/ps/astro-ph/0304163v1 
This figure "figure13a.png" is available in "png" format from: http://arxiv.org/ps/astro-ph/0304163v1 
This figure "figure13b.png" is available in "png" format from: http://arxiv.org/ps/astro-ph/0304163v1 
This figure "figure14a.png" is available in "png" format from: http://arxiv.org/ps/astro-ph/0304163v1 
This figure "figure14b.png" is available in "png" format from: http://arxiv.org/ps/astro-ph/0304163v1 
This figure "figure15.png" is available in "png" format from: http://arxiv.org/ps/astro-ph/0304163v1 
This figure "figure16.png" is available in "png" format from: http://arxiv.org/ps/astro-ph/0304163v1 
This figure "figure17.png" is available in "png" format from: http://arxiv.org/ps/astro-ph/0304163v1 
This figure "figure18a.png" is available in "png" format from: http://arxiv.org/ps/astro-ph/0304163v1 
This figure "figure18b.png" is available in "png" format from: http://arxiv.org/ps/astro-ph/0304163v1 
This figure "figure19a.png" is available in "png" format from: http://arxiv.org/ps/astro-ph/0304163v1 
This figure "figure19b.png" is available in "png" format from: http://arxiv.org/ps/astro-ph/0304163v1 